\title{
Scrotal Abscess in a Japanese Patient Caused by Prevotella bivia and Streptococcus agalactiae, Successfully Treated with Cefazolin and Amoxicillin: A Case Report
}

\author{
Haruka Watanabe \\ Yuta Norimatsu (D) ${ }^{1,2}$ \\ Yuki Ohno' \\ 'Department of Dermatology, JR Tokyo \\ General Hospital, Tokyo, Japan; \\ ${ }^{2}$ Department of Dermatology, University \\ of Tokyo Graduate School of Medicine, \\ Tokyo, Japan
}

Correspondence: Yuta Norimatsu JR Tokyo General Hospital, 2-I-3 Yoyogi Shibuya-ku, Tokyo, I5I-8528, Japan

Tel +8I-3-3320-2210

Fax +8I-3-3370-7478

Email norimatsuy-der@h.u-tokyo.ac.jp
Background: Infections caused by Prevotella bivia, a gram-negative anaerobic bacillus, are rare, with no reported cases in Japan. We present a novel case of scrotal abscess in a Japanese patient co-infected with Prevotella bivia and Streptococcus agalactiae.

Case Presentation: A 41-year-old uncontrolled diabetic man complained of swelling and pain in the scrotum. On examination, computed tomography revealed an abscess of 5-cm diameter in the scrotum. Then, the abscess was incised and drained. He was treated with cefazolin empirically. Prevotella bivia and Streptococcus agalactiae were identified in the pus cultures obtained from the abscess. However, the susceptibility tests for Prevotella bivia could not be submitted. Seven days following admission, the pain reduced, and the drainage slowed. The patient was discharged on day 14 when cefazolin was discontinued and oral amoxicillin (750 mg/day) was started. Amoxicillin was continued until day 42; improvement was confirmed. Conclusion: To the best of our knowledge, this case is the first report of Prevotella bivia in Japan. We suggest that cephem antibiotics such as cefazolin may be effective against Prevotella bivia in Japan.

Keywords: case report, Prevotella bivia, Streptococcus agalactiae, cefazolin, amoxicillin

\section{Background}

Approximately $90 \%$ of skin infections are caused by Staphylococcus aureus and Streptococcus pyogenes, sometimes in combination with anaerobic bacteria such as Bacteroides and Prevotella species. ${ }^{1,2}$ Prevotella species are present in the vaginal flora and can cause pelvic inflammatory diseases along with perirectal abscess. ${ }^{3,4}$ Prevotella bivia is a gram-negative anaerobic bacillus whose virulence is presumably enhanced by low oxygen tension in a polymicrobial environment. ${ }^{5}$ Prevotella bivia infections in humans are rare, with only 18 published cases in 44 years. $^{6-23}$ Since most Prevotella bivia strains are $\beta$-lactamase-positive, ${ }^{24}$ clindamycin, amoxicillin/clavulanate, metronidazole, and imipenem are the recommended antibacterial agents. $^{21,25}$ However, antibiotic resistance varies across countries, ${ }^{26}$ and it is unknown whether Prevotella bivia strains in Japan are $\beta$-lactamase positive.

To the best of our knowledge, there have been no reported cases of Prevotella bivia infections in Japan. Herein, we present a case of scrotal abscess in a Japanese patient that was caused by Prevotella bivia and Streptococcus agalactiae, and successfully treated using cefazolin followed by amoxicillin. 


\section{Case Presentation}

A 41-year-old man visited the urology department of JR Tokyo General Hospital with complaints of swelling and pain in the scrotum that had commenced 3 days earlier. A urologist diagnosed epididymitis and prescribed levofloxacin at $500 \mathrm{mg} /$ day. However, the symptoms did not improve, and the patient visited the dermatology department of JR Tokyo General Hospital (day 0).

On admission, he presented with a temperature of $36.5^{\circ} \mathrm{C}$, a blood pressure of $131 / 80 \mathrm{mmHg}$, and a heart rate of 100 beats per minute. His height and weight were $164 \mathrm{~cm}$ and $94.8 \mathrm{~kg}$, respectively (body mass index: $35.2 \mathrm{~kg} / \mathrm{m}^{2}$ ). Local examination revealed that the scrotum was markedly swollen. The laboratory workup on day 0 revealed elevated levels of serum C-reactive protein $(6.71 \mathrm{mg} / \mathrm{dL}$ ) (normal: $\leq 0.30 \mathrm{mg} / \mathrm{dL}$ ) and white blood cells $(13,800 / \mu \mathrm{L})$ (normal: $\leq 8600 \mathrm{mg} / \mathrm{dL}$ ). Furthermore, untreated diabetes was diagnosed owing to high hemoglobin Alc level (11.5\%) (normal: $\leq 6.0 \%$ ), and insulinenhancing therapy was initiated on day 0 . The patient's anti-streptolysin $\mathrm{O}$ antibody titer was $49 \mathrm{U} / \mathrm{mL}$ (normal: $\leq 239 \mathrm{U} / \mathrm{mL}$ ), and no renal or hepatic dysfunction was observed. Test results for syphilis (rapid plasma reagin and Treponema pallidum hemagglutination) and human immunodeficiency virus antibodies were negative. The patient had not visited a medical institution for about 20 years; therefore, he declared no medical history. His partner was a woman, and he did not engage in commercial sex. Notably, there was no family history.

Additionally, computed tomography revealed an abscess with a diameter of $5 \mathrm{~cm}$ in the scrotum without gas image (Figure 1). There were no abnormal findings in the testes or epididymis. On day 0 , the skin above the abscess was incised, which was drained, and the pus obtained was submitted for culture. We do not have a dedicated transport spitz for anaerobic bacteria. Therefore, we contacted the bacterial laboratory before the incision and asked them to prepare the culture in advance. About $5 \mathrm{cc}$ of pus was put into a sterilized spitz and transported to the bacterial laboratory within a few minutes for immediate culturing. Additionally, the blood culture was submitted on day 0 . Intravenous administration of cefazolin $(2 \mathrm{~g} / 8 \mathrm{~h})$ was empirically initiated as an antibacterial treatment for the scrotal abscess. The blood culture was negative. Culturing of the pus in the abscess revealed the presence of Prevotella spp. (RapID ANA II System, Thermo Fisher Scientific, United States) and

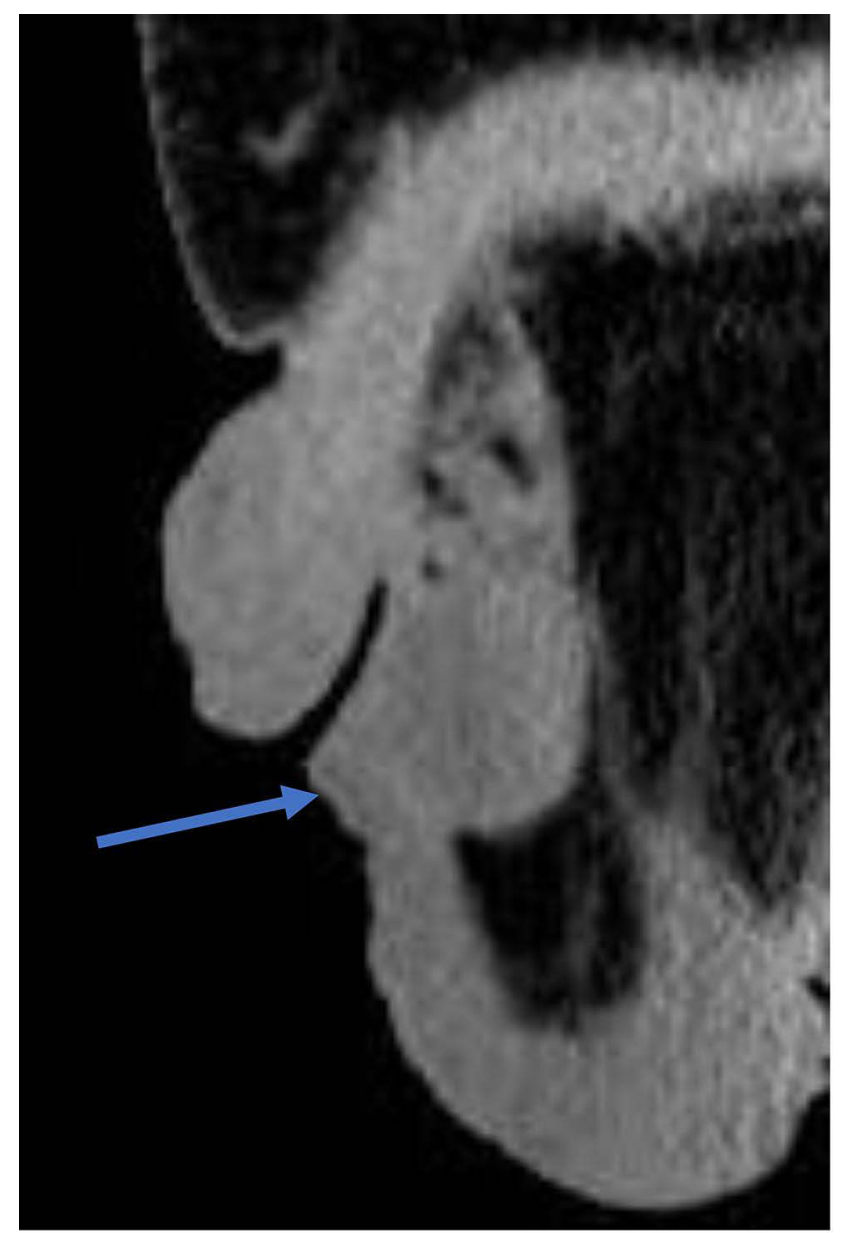

Figure I Computed tomography revealed an abscess with a diameter of $5 \mathrm{~cm}$ in the scrotum without gas image (sagittal section).

Streptococcus agalactiae (group B) spp. (Prolex ${ }^{\mathrm{TM}}$ Streptococcal Grouping Latex Kit, IWAKI\&CO., LTD., Japan). Furthermore, the antibiotic susceptibility pattern of Streptococcus agalactiae was done according to MICroFAST 7J, BECKMAN COULTER, United States; therefore, intravenous administration of cefazolin was empirically continued (Table 1). In Japan, the antibiotic susceptibility of Prevotella bivia could not be measured this time because it can be measured only at a specific research facility.

Although the symptoms exhibited improvements, pus drainage continued, and the pain persisted; therefore, a second pus culture was performed on day 7. A large number of gram-negative bacilli were observed and identified as Prevotella bivia (RapID ANA II System, Thermo Fisher Scientific, United States) on day 10 (the pus culture was negative for Streptococcus agalactiae). At this time, the pain subsided, and the pus drainage slowed. The patient was discharged on day 14 when cefazolin was 
Table I Antibacterial Sensitivity Test (MICroFAST 7J, BECKMAN COULTER, United States) Results for Streptococcus agalactiae Cultured from the Pus Collected from the Patient's Scrotal Abscess

\begin{tabular}{|l|l|l|}
\hline Drug & MIC & Interpretation \\
\hline Ampicillin & 0.12 & Sensitive \\
Penicillin G & 0.06 & Sensitive \\
Amoxicillin/clavulanate & $\leq 0.25$ & Not assessed \\
Cefotiam & $\leq 0.5$ & Not assessed \\
Cefotaxime & $\leq 0.12$ & Sensitive \\
Ceftriaxone & $\leq 0.12$ & Sensitive \\
Cefepime & $\leq 0.5$ & Sensitive \\
Cefditoren pivoxil & $\leq 0.06$ & Not assessed \\
Cefozopran & $\leq 0.12$ & Sensitive \\
Meropenem & $\leq 0.12$ & Sensitive \\
Erythromycin & $\leq 0.12$ & Sensitive \\
Clindamycin & $\leq 0.12$ & Sensitive \\
Minocycline & $\leq 0.5$ & Sensitive \\
Vancomycin & 0.5 & Sensitive \\
Chloramphenicol & $\leq 4$ & Sensitive \\
Rifampicin & $\leq 1$ & Not assessed \\
Sulfamethoxazole/trimethoprim & $\leq 0.5$ & Not assessed \\
Levofloxacin & $>8$ & Resistant \\
Azithromycin & $\leq 0.25$ & Sensitive \\
\hline
\end{tabular}

Abbreviation: MIC, minimum inhibitory concentration $(\mu \mathrm{g} / \mathrm{mL})$.

discontinued and oral amoxicillin $(750 \mathrm{mg} / \mathrm{day})$ was commenced. In addition, the patient visited the hospital every week. We determined the need to continue antibiotics based on clinical symptoms. Amoxicillin was continued until day 42 , and improvement was clinically confirmed.

\section{Discussion and Conclusions}

We encountered a case of scrotal abscess due to Prevotella bivia and Streptococcus agalactiae. Prevotella bivia is predominantly associated with low-grade infections in the female urogenital tract, presenting as endometritis, pelvic inflammatory disease, or perirectal or anal abscess. ${ }^{3,4,27,28}$ However, it can also cause infections in non-gynecological tissues.

Table 2 summarizes previous reports of Prevotella bivia infections as published in PubMed. ${ }^{6-23}$ Similar to our case, the case presented by Bekasiak et al involved a scrotal abscess and co-infection with a second bacterial genus. ${ }^{11}$ Additionally, co-infection occurred in 7 cases, including 2 cases with Prevotella bivia and Streptococcus anginosus, a well-known species that produces an abscess. ${ }^{2}$ The presence of co-infectious bacteria is important for Prevotella bivia infections.
In particular, when Prevotella bivia and Streptococcus agalactiae are co-infectious, they reportedly have a $100 \%$ chance of causing infection in a mouse model. ${ }^{5}$ Knowledge of other bacterial genera present is important when treating Prevotella bivia infections, since they may alter the pathogenicity of Prevotella bivia. ${ }^{29}$ Abscesses occurred in seven of the 19 cases, including our case. The presence of abscess formation in approximately $37 \%$ of reported cases of Prevotella bivia infection is considered a non-negligible finding. Prevotella bivia infection should also be kept in mind when the patient has an abscess. In many cases shown in Table 2, metronidazole or clindamycin was the chosen treatment. Prevotella bivia is susceptible to these drugs, as well as to amoxicillin/clavulanate and imipenem. ${ }^{21,25}$ However, our patient was successfully treated with cefazolin followed by oral amoxicillin. There are two possible reasons for this. First, the mixed infection by facultative anaerobe pathogen Streptococcus agalactiae is the factor that lowers the tissue oxygen tension and allows for the growth of anaerobic Prevotella bivia after eradication of the Streptococcus agalactiae, which was evidenced. ${ }^{5}$ Second, Prevotella bivia strains in Japan may be sensitive to cefazolin and amoxicillin, whereas strains in other countries are resistant. In support of cefazolin sensitivity, a similar antibiotic (flomoxef) has exhibited effectiveness against Prevotella bivia in an experimental animal model in Japan. ${ }^{5}$ Unfortunately, there was no drug susceptibility test for Prevotella bivia conducted in our case; thus, whether it was susceptible to cefazolin or amoxicillin is unknown.

Among the 19 patients in Table 2, five had no medical history, and 17 were 65 years old or younger. Thus, immunodeficiency and aging may not be risk factors for Prevotella bivia infection, as they are for cellulitis. ${ }^{30}$ In Table 2, diabetes or obesity was observed in four of the 19 cases. Moreover, diabetes is common among Japanese people and thus may be a risk factor for Prevotella bivia infection. ${ }^{30}$

In conclusion, we encountered a case of scrotal abscess caused by Prevotella bivia and Streptococcus agalactiae in a Japanese patient. To the best of our knowledge, there have been no reported cases of Prevotella bivia infections in Japan. Therefore, we suggest that cephem antibiotics such as cefazolin may effectively treat Prevotella bivia in Japanese patients if incision and drainage are properly performed. 
Table 2 Summary of Previous Studies of Prevotella bivia Infections

\begin{tabular}{|c|c|c|c|c|c|c|c|}
\hline \multirow[t]{2}{*}{ Author } & \multirow[t]{2}{*}{ Diagnosis } & Age & \multirow[t]{2}{*}{ Co-Infection } & \multirow[t]{2}{*}{ Medical History } & \multirow[t]{2}{*}{ First Therapy } & \multirow[t]{2}{*}{ Second Therapy } & \multirow{2}{*}{$\begin{array}{l}\text { Third } \\
\text { Therapy }\end{array}$} \\
\hline & & Sex & & & & & \\
\hline \multirow{2}{*}{$\begin{array}{l}\text { Grande- } \\
\text { Del-Arco }\end{array}$} & \multirow[t]{2}{*}{ Paronychia } & 38 & \multirow{2}{*}{$\begin{array}{l}\text { Staphylococcus } \\
\text { haemolyticus }\end{array}$} & \multirow[t]{2}{*}{ Onychomycosis } & \multirow{2}{*}{$\begin{array}{l}\text { Metronidazole } \\
(500 \mathrm{mg}), \\
\text { cephalexin } \\
(500 \mathrm{mg}), \text { every } \\
8 \mathrm{~h} \text { per os }\end{array}$} & \multirow{2}{*}{$\begin{array}{l}\text { Moxifloxacin (400 mg/ } \\
\text { day for } 2 \text { weeks) }\end{array}$} & \multirow{2}{*}{$\begin{array}{l}\text { Rifampicin } \\
(600 \mathrm{mg} / \\
\text { day })\end{array}$} \\
\hline & & $\mathrm{F}$ & & & & & \\
\hline \multirow[t]{2}{*}{ Boucher } & \multirow{2}{*}{$\begin{array}{l}\text { Nonpuerperal } \\
\text { breast abscess }\end{array}$} & 39 & \multirow[t]{2}{*}{ None } & \multirow{2}{*}{$\begin{array}{l}\text { IgA nephropathy } \\
\text { (Berger's disease), } \\
\text { hypertension }\end{array}$} & \multirow{2}{*}{$\begin{array}{l}\text { Flucloxacillin } \\
(500 \mathrm{mg} / 6 \mathrm{~h})\end{array}$} & \multirow{2}{*}{$\begin{array}{l}\text { Amoxicillin/clavulanate } \\
(625 \mathrm{mg} / 8 \mathrm{~h}), \\
\text { metronidazole }(500 \mathrm{mg} \\
8 \mathrm{~h})\end{array}$} & \multirow[t]{2}{*}{ None } \\
\hline & & $\mathrm{F}$ & & & & & \\
\hline \multirow[t]{2}{*}{ Mohan } & \multirow{2}{*}{$\begin{array}{l}\text { Renal and } \\
\text { perinephric } \\
\text { abscesses }\end{array}$} & 26 & \multirow[t]{2}{*}{ Lactobacillus jensenii } & \multirow[t]{2}{*}{ Stable renal cyst } & \multirow{2}{*}{$\begin{array}{l}\text { Cefepime, } \\
\text { vancomycin }\end{array}$} & \multirow{2}{*}{$\begin{array}{l}\text { Ceftriaxone }(2 \mathrm{~g} / \text { day }) \text {, } \\
\text { metronidazole }(500 \mathrm{mg} / \\
8 \mathrm{~h})\end{array}$} & \multirow[t]{2}{*}{ None } \\
\hline & & $\mathrm{F}$ & & & & & \\
\hline \multirow[t]{2}{*}{ Samantara } & \multirow{2}{*}{$\begin{array}{l}\text { Intracranial } \\
\text { abscess }\end{array}$} & 50 & \multirow{2}{*}{$\begin{array}{l}\text { Methicillin- resistant } \\
\text { Staphylococcus aureus }\end{array}$} & \multirow[t]{2}{*}{ Head trauma } & \multirow[t]{2}{*}{ Metronidazole } & None & None \\
\hline & & M & & & & & \\
\hline Bekasiak & Scrotal abscess & 27 & Gardnerella vaginalis & Obesity & Ceftriaxone (I g/ & Cephalexin (500 mg P.O., & None \\
\hline & & M & & & day) & $\begin{array}{l}4 \text { times/day), } \\
\text { metronidazole ( } 500 \mathrm{mg}, 3 \\
\text { times/day) }\end{array}$ & \\
\hline Kostov & Fulminant & 39 & None & None & Gentamicin & Metronidazole $(500 \mathrm{mg}$ & None \\
\hline & $\begin{array}{l}\text { generalized } \\
\text { peritonitis }\end{array}$ & $\mathrm{F}$ & & & $\begin{array}{c}\text { (120 mg/I2 h, i. } \\
\text { m.), cefazolin ( } 2 \\
\text { g every I } 2 \text { h, i.v.), } \\
\text { metronidazole } \\
\text { (500 mg every } 8 \\
\text { h, i.v.) }\end{array}$ & every 8 h, i.v.) & \\
\hline Masadeh & Purulent & 32 & None & None (homosexual) & Ceftriaxone & Doxycycline, & None \\
\hline & proctitis & $M$ & & & & metronidazole & \\
\hline Di Marco & Empyema & 78 & None & Chronic obstructive & Levofloxacin & Intravenous clindamycin & Oral \\
\hline Berardino & & M & & $\begin{array}{l}\text { pulmonary disease, } \\
\text { chronic periodontitis }\end{array}$ & (750 mg/day) & $(600$ mg/8 h) & $\begin{array}{c}\text { clindamycin } \\
\text { (300 mg/6 } \\
\text { h for } 4 \\
\text { weeks) }\end{array}$ \\
\hline Mirza & Paronychia & 17 & Methicillin-sensitive & None & Cefazolin & Oral ciprofloxacin & None \\
\hline & & $\mathrm{F}$ & Staphylococcus aureus & & & & \\
\hline Mirza & Paronychia & 55 & Enterococcus spp., & None & Oral & Oral clindamycin & Oral \\
\hline & & $\mathrm{F}$ & $\begin{array}{l}\text { Pseudomonas aeruginosa } \\
\text { and melaninogenica }\end{array}$ & & ciprofloxacin & & ciprofloxacin \\
\hline Janssen & Abdominal & 55 & None & Diabetes mellitus, renal & Metronidazole & Metronidazole, & None \\
\hline & wall phlebitis & $\mathrm{F}$ & & $\begin{array}{c}\text { transplantation, } \\
\text { haemodialysis, obesity }\end{array}$ & & ceftriaxone & \\
\hline
\end{tabular}

(Continued) 
Table 2 (Continued).

\begin{tabular}{|c|c|c|c|c|c|c|c|}
\hline \multirow[t]{2}{*}{ Author } & \multirow[t]{2}{*}{ Diagnosis } & Age & \multirow[t]{2}{*}{ Co-Infection } & \multirow[t]{2}{*}{ Medical History } & \multirow[t]{2}{*}{ First Therapy } & \multirow[t]{2}{*}{ Second Therapy } & \multirow{2}{*}{$\begin{array}{c}\text { Third } \\
\text { Therapy }\end{array}$} \\
\hline & & Sex & & & & & \\
\hline \multirow[t]{2}{*}{ Lepivert } & \multirow{2}{*}{$\begin{array}{l}\text { Necrotizing } \\
\text { fasciitis }\end{array}$} & 65 & \multirow[t]{2}{*}{ None } & \multirow[t]{2}{*}{ Diabetes mellitus } & \multirow{2}{*}{$\begin{array}{c}\text { Intravenous } \\
\text { vancomycin }(2 \mathrm{~g} / \\
24 \mathrm{~h}) \text {, } \\
\text { piperacillin/ } \\
\text { tazobactam }(4 \mathrm{~g} / \\
\mathrm{h})\end{array}$} & \multirow{2}{*}{$\begin{array}{l}\text { Intravenous amoxicillin- } \\
\text { clavulanic acid }(\lg / 6 \mathrm{~h}) \text {, } \\
\text { levofloxacin }(500 \mathrm{mg} / 12 \\
\text { h) }\end{array}$} & \multirow{2}{*}{$\begin{array}{c}\text { Oral } \\
\text { amoxicillin/ } \\
\text { clavulanic } \\
\text { acid, } \\
\text { levofloxacin }\end{array}$} \\
\hline & & $\mathrm{F}$ & & & & & \\
\hline \multirow[t]{2}{*}{ Hsu } & \multirow{2}{*}{$\begin{array}{l}\text { Chest wall } \\
\text { abscess }\end{array}$} & 77 & \multirow[t]{2}{*}{ None } & \multirow[t]{2}{*}{ None } & \multirow{2}{*}{$\begin{array}{l}\text { Oral amoxicillin/ } \\
\text { clavulanate }\end{array}$} & \multirow[t]{2}{*}{ None } & \multirow[t]{2}{*}{ None } \\
\hline & & M & & & & & \\
\hline \multirow[t]{2}{*}{ Nalmas } & \multirow[t]{2}{*}{ Penile abscess } & 44 & \multirow[t]{2}{*}{ Streptococcus constellatus } & \multirow{2}{*}{$\begin{array}{c}\text { Hypertension, } \\
\text { obstructive sleep apnea, } \\
\text { mild asthma }\end{array}$} & \multirow{2}{*}{$\begin{array}{l}\text { Intravenous } \\
\text { vancomycin, } \\
\text { clindamycin, } \\
\text { cefepime }\end{array}$} & \multirow{2}{*}{$\begin{array}{l}\text { Intravenous } \\
\text { vancomycin, } \\
\text { clindamycin }\end{array}$} & \multirow{2}{*}{$\begin{array}{c}\text { Oral } \\
\text { amoxicillin/ } \\
\text { clavulanate } \\
\text { (875 mg, } \\
\text { twice/day) }\end{array}$} \\
\hline & & M & & & & & \\
\hline \multirow[t]{2}{*}{ Huits } & \multirow{2}{*}{$\begin{array}{l}\text { Lemierre's } \\
\text { syndrome }\end{array}$} & 17 & \multirow[t]{2}{*}{ None } & \multirow[t]{2}{*}{ None } & \multirow{2}{*}{$\begin{array}{c}\text { Intravenous } \\
\text { amoxicillin/ } \\
\text { clavulanic acid } \\
\text { (1000/200 mg } \\
\text { tds) }\end{array}$} & \multirow{2}{*}{$\begin{array}{l}\text { Benzyl-penicillin } \\
\text { (6 million units tds IV), } \\
\text { metronidazole ( } 500 \mathrm{mg} \\
\text { qid IV) }\end{array}$} & \multirow[t]{2}{*}{ None } \\
\hline & & $\mathrm{F}$ & & & & & \\
\hline \multirow[t]{2}{*}{ Riesbeck } & Paronychia & 45 & $\beta$-hemolytic & Adiposity, non-insulin- & |soxazoly| & Intravenous cefuroxime, & Oral \\
\hline & & M & $\begin{array}{c}\text { streptococci group B, } \\
\text { Streptococcus milleri } \\
\text { group }\end{array}$ & $\begin{array}{c}\text { dependent diabetes } \\
\text { mellitus }\end{array}$ & penicillin & metronidazole & clindamycin \\
\hline Laiho & Septic arthritis & 23 & None & Juvenile rheumatoid & Imipenem & None & None \\
\hline & & $\mathrm{F}$ & & arthritis & (1000 mg) & & \\
\hline Sagristà & Inguinal & 34 & None & None (unprotected & Doxycycline, & Oral amoxicillin/ & None \\
\hline & syndrome & M & & $\begin{array}{l}\text { sexual intercourse with } \\
\text { a woman) }\end{array}$ & ciprofloxacin & clavulonate & \\
\hline Our case & Scrotal abscess & 41 & Streptococcus agalactiae & Diabetes mellitus & Levofloxacin & Cefazolin & Oral \\
\hline & & M & & & & & amoxicillin \\
\hline
\end{tabular}

Abbreviations: F, female; M, male.

\section{Ethics Approval and Consent to Participate}

Submission of this manuscript has been approved by the JR Tokyo General Hospital's ethics review board.

\section{Consent for Publication}

We obtained written signed informed consent from the patient to publish his clinical details.

\section{Informed Consent}

We obtained written signed consent from the patient to publish his clinical details.

\section{Acknowledgments}

We would like to thank Editage (www.editage.jp) for English language editing.

\section{Author Contributions}

All authors contributed to data analysis, drafting or revising the article, gave final approval of the version to be published, agreed to the submitted journal, and agree to be accountable for all aspects of the work.

\section{Funding}

There is no funding to report. 


\section{Disclosure}

The authors report no conflicts of interest in this work.

\section{References}

1. Jeng A, Beheshti M, Li J, Nathan R. The role of beta-hemolytic streptococci in causing diffuse, nonculturable cellulitis: a prospective investigation. Medicine (Baltimore). 2010;89 (4):217-226. doi:10.1097/MD.0b013e3181e8d635

2. Asam D, Spellerberg B. Molecular pathogenicity of Streptococcus anginosus. Mol Oral Microbiol. 2014;29(4):145-155. doi:10.1111/ omi.12056

3. Brook I, Frazier EH. The aerobic and anaerobic bacteriology of perirectal abscesses. J Clin Microbiol. 1997;35(11):2974-2976. doi:10.1128/jcm.35.11.2974-2976.1997

4. Gossling J. Occurrence and pathogenicity of the Streptococcus milleri group. Rev Infect Dis. 1988;10(2):257-285. doi:10.1093/clinids/10.2.257

5. Mikamo H, Kawazoe K, Izumi K, Watanabe K, Ueno K, Tamaya T. Studies on the pathogenicity of anaerobes, especially Prevotella bivia, in a rat pyometra model. Infect Dis Obstet Gynecol. 1998;6(2):61. doi:10.1002/(SICI)1098-0997(1998)6:2<61::AID-IDOG6>3.0.CO;2-A

6. Holdeman LV, Johnson JL. Bacteroides disiens sp. nov. and Bacteroides bivius sp. nov. from human clinical infections. Int $J$ Syst Bacteriol. 1977;27:337-345. doi:10.1099/00207713-27-4-337

7. Grande-Del-Arco J, Jimenez-Cristino MD, García-de-la-peña R, Fernández-Espejo E, Córdoba-Fernández A, Rare A. Paronychia with superinfection with Prevotella bivia and Staphylococcus haemolyticus: the importance of early microbiological diagnosis. Pathogens. 2020;9(12):999. doi:10.3390/pathogens9120999

8. Boucher A, Quaranta D, Emonet S, Serratrice J, Coen M. Nonpuerperal breast abscess due to Prevotella bivia. Clin Case Rep. 2020;8(8):1399-1402. doi:10.1002/ccr3.2824

9. Mohan A, Rubin J, Chauhan P, Ramirez JL, Giese G. Renal and perinephric abscesses involving Lactobacillus jensenii and Prevotella bivia in a young woman following ureteral stent procedure. $J$ Commun Hosp Intern Med Perspect. 2020;10(2):162-165. doi:10.1080/20009666.2020.1742494

10. Samantaray S, Biswas R, Sasidharan GM. Intracranial abscess due to Prevotella bivia: first case report from India. Anaerobe. 2020;65:102249. doi:10.1016/j.anaerobe.2020.102249

11. Bekasiak A, Dammann F, Nader C, Rare A. Cause of a scrotal abscess due to the symbiotic infection of Gardnerella vaginalis and Prevotella bivia in an adult male. Pathogens. 2020;9(2):93. doi:10.3390/pathogens 9020093

12. Kostov S, Slavchev S, Dzhenkov D, Strashilov S, Yordanov A. An unusual case of fulminant generalized peritonitis secondary to purulent salpingitis caused by Prevotella bivia - case report with literature review. GERMS. 2020;10(1):51-54. doi:10.18683/germs.2020.1185

13. Masadeh M, Hossain S, Dunkelberg J, Gerke H. Purulent proctitis caused by Prevotella bivia in a homosexual male. ACG Case Rep J. 2016;3(4):e178. doi:10.14309/crj.2016.151

14. Di Marco Berardino A, Inchingolo R, Smargiassi A, et al. Empyema caused by Prevotella bivia complicating an unusual case of spontaneous chylothorax. $J$ Clin Microbiol. 2014;52(4):1284-1286. doi:10.1128/JCM.03282-13
15. Mirza A, Bove JJ, Litwa J, Appelbe G. Mixed infections of the paronychium with Prevotella bivia. J Hand Microsurg. 2012;4 (2):77-80. doi:10.1007/s12593-011-0054-7

16. Janssen S, van Donselaar-van der Pant KA, van der Weerd NC, et al. Abdominal wall phlebitis due to Prevotella bivia following renal transplantation in a patient with an occluded inferior vena cava. Infection. 2013;41(1):271-274. doi:10.1007/s15010-012-0335-5

17. Lepivert JC, Guinet V, Auquit-Auckbur I. Necrotizing fasciitis of the hand and wrist due to Prevotella bivia. J Hand Surg Eur. 2015;40 (7):757-758. doi:10.1177/1753193414545942

18. Hsu GJ, Chen CR, Lai MC, Luh SP. Chest wall abscess due to Prevotella bivia. J Zhejiang Univ Sci B. 2009;10(3):233-236. doi:10.1631/jzus.B0820289

19. Nalmas S, Bishburg E, Chan T. Streptococcus constellatus and Prevotella bivia penile abscess. Scientific World J. 2007;7:1631-1633. doi:10.1100/tsw.2007.240

20. Huits RM, van Assen S, Wildeboer-Veloo AC, Verschuuren EA, Koeter GH. Prevotella bivia necrobacillosis following infectious mononucleosis. $J$ Infect. 2006;53(2):e59-e63. doi:10.1016/j.jinf.2005.10.016

21. Riesbeck K. Paronychia due to Prevotella bivia that resulted in amputation: fast and correct bacteriological diagnosis is crucial. J Clin Microbiol. 2003;41(10):4901-4903. doi:10.1128/JCM.41.10.4901-4903.2003

22. Laiho K, Kotilainen P. Septic arthritis due to Prevotella bivia after intra-articular hip joint injection. Joint Bone Spine. 2001;68 (5):443-444. doi:10.1016/S1297-319X(01)00303-7

23. Sagristà M, Martin-Ezquerra G, Gallardo F, et al. Inguinal syndrome secondary to Prevotella bivia after accidental bite in orogenital sex. Sex Transm Infect. 2012;88(4):250-251. doi:10.1136/sextrans-2011-050348

24. Wybo I, Piérard D, Verschraegen I, et al. Third Belgian multicentre survey of antibiotic susceptibility of anaerobic bacteria. J Antimicrob Chemother. 2007;59(1):132-139. doi:10.1093/jac/dk1458

25. Stein GE, Schooley S, Tyrrell KL, Citron DM, Goldstein EJ. Human serum activity of telithromycin, azithromycin and amoxicillin/clavulanate against common aerobic and anaerobic respiratory pathogens. Int $J$ Antimicrob Agents. 2007;29(1):39-43. doi:10.1016/j. ijantimicag.2006.08.041

26. Johnson AP, Davies J, Guy R, et al. Mandatory surveillance of methicillin-resistant Staphylococcus aureus (MRSA) bacteraemia in England: the first 10 years. J Antimicrob Chemother. 2012;67 (4):802-809. doi:10.1093/jac/dkr561

27. Jousimies-Somer H. Recently described clinically important anaerobic bacteria: taxonomic aspects and update. Clin Infect Dis. 1997;25 (Suppl 2):S78-S87. doi:10.1086/516227

28. Strömbeck L, Sandros J, Holst E, et al. Prevotella bivia can invade human cervix epithelial (HeLa) cells. APMIS. 2007;115(3):241-251. doi:10.1111/j.1600-0463.2007.apm_512.x

29. Brook I. The role of anaerobic bacteria in tonsillitis. Int $J$ Pediatr Otorhinolaryngol. 2005;69(1):9-19. doi:10.1016/j.ijporl.2004.08.007

30. Norimatsu Y, Ohno Y. Predictors for readmission due to cellulitis among Japanese patients. J Dermatol. 2021;48(5):681-684. doi:10.1111/1346-8138.15771 


\section{Publish your work in this journal}

The International Medical Case Reports Journal is an international, peer-reviewed open-access journal publishing original case reports from all medical specialties. Previously unpublished medical posters are also accepted relating to any area of clinical or preclinica science. Submissions should not normally exceed 2,000 words or 4 published pages including figures, diagrams and references. The manuscript management system is completely online and includes a very quick and fair peer-review system, which is all easy to use. Visit http://www.dovepress.com/testimonials.php to read real quotes from published authors. 